This is an author-created, un-copyedited version of an article accepted for publication in Journal of Breath Research. IOP Publishing Ltd is not responsible for any errors or omissions in this version of the manuscript or any version derived from it. The definitive publisher-authenticated version is available online at https:// doi.org/10.1088/1752-7155/10/4/046002. 
The use of spirometry filters as a novel sampling device for influenza

\section{and other viruses}

Alicia B. Mitchell, BMedSc(Hons) $)^{1,2}$, Euan Tovey, $\mathrm{PhD}^{2}$, Lachlan Buddle, BMedSc ${ }^{3}$, Matthew Peters, $\mathrm{MBBS}^{3}$, Lucy Morgan, $\mathrm{MBBS}^{3}$, and Brian G. Oliver, $\mathrm{PhD}^{2,4}$.

${ }^{1}$ Discipline of Pharmacology, Faculty of Medicine

The University of Sydney

NSW, 2006, Australia

${ }^{2}$ Woolcock Institute of Medical Research

The University of Sydney

NSW, 2006, Australia

${ }^{3}$ Department of Respiratory Medicine

Concord Repatriation General Hospital

Concord, NSW, 2139, Australia

${ }^{4}$ School of Medical and Molecular Biosciences

University of Technology Sydney

NSW, 2007, Australia

$\begin{array}{ll}\text { Corresponding author: } & \text { Alicia B. Mitchell } \\ & \text { Woolcock Institute of Medical Research } \\ & \text { 431 Glebe Point Road } \\ & \text { Glebe, NSW, 2037, Australia } \\ & +61291140302 \\ \text { Phone: } & +61291140399 \\ \text { Fax: } & \text { amit9422@uni.sydney.edu.au } \\ \text { Email: } & \end{array}$


Summary at a glance: This study investigated a novel viral sampling technique in comparison to currently used methods. It was found that spirometry mouthpiece filters are useful in capturing and PCR detection of common respiratory viruses. This may allow viral testing to become more commonplace in routine clinical practice.

\section{Abstract:}

Aim: Respiratory viruses contribute to the burden of illness for patients with chronic respiratory disease. Many studies have reported a high prevalence of viruses in patients experiencing exacerbations. These studies use sampling methods that are invasive, difficult to collect, and not ideal for repetitive measurements. The aim of this study was to explore whether respiratory viruses could be isolated from disposable spirometry filters.

Methods: Patients with chronic obstructive pulmonary disease (COPD), asthma, bronchiectasis and healthy controls were recruited from June to September 2014. Patients completed spirometry and provided a nasal washing or sputum sample. These, together with eluate from the spirometry filters were examined using reverse transcription polymerase chain reaction (PCR) to identify respiratory viruses.

Results: 53 patients were recruited (COPD $n=13$; asthma $n=17$; bronchiectasis $n=13$ ) including 10 healthy controls $(n=10)$. One or more virus was detected in any sample in $83 \%$ of participants, and was identified in the spirometry filters in $56 \%$ of participants. Of these, $81 \%$ had the same virus type seen in sputum and/or nasal washings. $77 \%$ of participants with COPD, $88 \%$ with asthma, and $92 \%$ with bronchiectasis tested viral positive in at least one sample compared with $70 \%$ of healthy controls. 
Conclusion: Virus identified in spirometry filters corresponded well with virus identified in sputum/nasal lavage. Overall, higher levels of virus were detected in patients with chronic respiratory disease than healthy controls. These results suggest that respiratory filters may be a novel and inexpensive sampling method for the presence of respiratory viruses in exhaled breath.

Short title: Use of exhaled breath to detect viruses

Keywords:

Asthma

Bronchiectasis

Influenza

Spirometry

Viruses 


\section{Introduction:}

Viral infections contribute significantly to the burden of chronic respiratory diseases. Many studies have indicated that viruses are precipitants of exacerbations ${ }^{(1,2)}$ since exacerbation frequency is strongly related to disease progression ${ }^{(3)}$. These viral infections can have a major impact on morbidity and mortality in these patients.

The WHO estimates influenza virus to cause 3 to 5 million cases of severe illness annually worldwide ${ }^{(4)}$. Influenza infects the lower respiratory tract by binding to sialic acid receptors and entering epithelial cells ${ }^{(5)}$. Symptoms include cough, sore throat, blocked nose, fever, headaches, general malaise and body aches in otherwise healthy individuals. However, in individuals with chronic diseases and compromised immune systems, this virus can cause complications such as severe pneumonia and death ${ }^{(6)}$. Early detection of viral infection may be crucial in mitigating the risk of developing complications.

Virus can be sampled from the respiratory tract in several ways. The most common sampling techniques for the upper respiratory tract include oropharyngeal or nasopharyngeal swabs and nasopharyngeal washings/aspirates. For the lower respiratory tract, secretions are sampled via induced or spontaneously expectorated sputum, and bronchoalveolar lavage. However, sensitive techniques such as nasopharyngeal aspirates and bronchoalveloar lavage ${ }^{(7-9)}$ are invasive and are not available in primary care or outpatient settings. There is also a lack of consistency in viral detection levels across studies as sensitivities reported for nasopharyngeal aspirates ranged from 67$97 \%{ }^{(9-11)}$. 
As many current methods for sampling lower respiratory secretions are invasive, sampling exhaled breath may offer a more desirable alternative ${ }^{(12)}$. Aerosol transmission appears to be an important method of spread, especially for influenza viruses ${ }^{(13)}$ and such detection may reflect replication and transmission peaks. Several different methods have been used to sample exhaled virus yet there is no standardised method or equipment.

The aim of our study was to investigate if spirometry filters could be used as a sampling device to collect exhaled viral aerosols, and compare the sensitivity of exhaled virus detection to sputum and nasal washing. 
Methods:

\section{Subjects}

Volunteers were recruited during routine clinic visits. Inclusion criteria for each chronic respiratory disease included having a clinician's diagnosis of COPD, Asthma or Bronchiectasis, being over 18 years of age and being able to complete lung function testing. Healthy controls also attended the clinic for reasons unrelated to a diagnosed respiratory disease. Ethics approval was obtained from the Sydney Local Health District Ethics committee at CRGH (study number CH62/6/2014-047). Prior to commencement of the study, written informed consent was obtained from all participants.

\section{Study Design}

This single-centre, cross sectional study was conducted from April until September 2014 in the Respiratory Department at Concord Repatriation General Hospital, Sydney, Australia. A total of 53 participants were included in this study. Participants were recruited within the outpatient respiratory lab when they attended routine lung function testing following a doctor's referral. All patients were clinically stable at the time of sampling. Healthy controls were also recruited during this time - these were patients with no previous history of lung disease who were referred for lung function testing for other reasons, such as follow up after surgery or due to an underlying cardiac problem..

All subjects performed at least three consecutive forced expiratory maneuvers and electret filters were collected from the spirometry mouthpieces (Bird Healthcare, Melbourne, Australia). Any spontaneously expectorated sputum was collected, otherwise nasal washings were collected. For nasal washing, subjects inhaled two 
sprays of saline into each nostril (approximately $0.1 \mathrm{ml}$ per spray), and then blew their nose into a plastic bag where the nasal lavage was collected. All samples were stored at $-80^{\circ} \mathrm{C}$ until processing. Subjects were asked questions regarding medications and general respiratory health. They were also asked to complete the common cold questionnaire (CCQ) previously described and evaluated by Powell et al (2008). This assesses symptoms over 4 categories - general, nasal, throat and chest symptoms, with individual domains considered in each category.

\section{Sample molecular processing}

Filters were first removed from the Spirometry mouthpieces and $1 \mathrm{ml}$ of Bioline Buffer RLY (Bioline, Alexandria, Australia) was then added. This was centrifuged for 2 minutes at $10,000 \mathrm{rpm}$. The eluate was collected after the final spin and stored at $-20^{\circ} \mathrm{C}$ until RNA extraction. Sputum and nasal washing samples were processed by mixing $0.2 \mathrm{~g}$ of secretion with $1 \mathrm{~mL}$ of Buffer RLY and were then stored at $-20^{\circ} \mathrm{C}$. Following this, RNA was purified using the Isolate RNA Mini Kit (Bioline, Alexandria, Australia) before conversion to cDNA using the Invitrogen M-MLV Reverse Transcriptase kit (Life Technologies, Mulgrave, Australia).

Spontaneously expectorated sputum, nasal washings and exhaled breath total cDNA was assayed by polymerase chain reaction (PCR) for human rhinovirus (hRV), respiratory syncytial virus (RSV), influenza virus type A and influenza virus type B.

The PCR assay utilised the Bioline PCR kit (Bioline, Alexandria, Australia) as follows: $4 \mu \mathrm{l}$ of cDNA template was added to $10 \mathrm{X}$ Buffer, $50 \mathrm{mM} \mathrm{MgCl}_{2}, 10 \mu \mathrm{M}$ Primers, $10 \mathrm{mM}$ dNTPs, $5 \mathrm{U}$ BioTaq Polymerase and DEPC $\mathrm{H}_{2} \mathrm{O}$ up to a total of $25 \mu 1$. The annealing 
temperature and number of cycles used were optimised for each primer set. Generalised PCR conditions were: $94^{\circ} \mathrm{C}$ for 2 minutes as the initial denaturation step, followed by 35 cycles ( 33 cycles for influenza B) of denaturation at $94^{\circ} \mathrm{C}$ for 30 seconds, 60 seconds of annealing at $60^{\circ} \mathrm{C}\left(55^{\circ} \mathrm{C}\right.$ for influenza B), extension at $72^{\circ} \mathrm{C}$ for 30 seconds; followed by a final extension step at $72^{\circ} \mathrm{C}$ for 5 minutes. PCR was carried out in an Eppendorf Mastercycler (Eppendorf South Pacific, North Ryde, Australia). As an internal control, positive viral cDNA was included in each PCR assay.

\section{Statistical Analysis}

GraphPad Prism version 6 was used to generate multivariate analysis of the raw nonadjusted dataset and this was used to investigate virus prevalence in the different diseases. 
$\underline{\text { Results: }}$

Over the three-month period, 53 participants agreed to be a part of this study. Of these, 13 patients had a clinical diagnosis of COPD, 17 of asthma, 13 of bronchiectasis and 10 were healthy controls.

\section{Virus detection}

The overall virus detection rates from the three sample types are shown in Figure 1. All participants provided a filter sample following spirometry testing; 58\% (31/53) of these samples were virus positive by PCR testing. Nasal washing or sputum samples were also provided by all participants, with 57\% (17/30) of nasal washing samples being viral positive and $87 \%(20 / 23)$ of sputum samples being viral positive. Sputum yielded the greatest rate of viral detection, and exhaled detection in these samples corresponded well (Table 1A). Nasal washing and exhaled samples still showed correspondence however this didn't agree as strongly as with sputum (Table 1B).

44 out of 53 participants $(83 \%)$ were viral positive with detection of at least one virus in either exhaled or respiratory secretion samples. $81 \%$ of positive viral detection in the participant's respiratory secretion sample correlated with exhaled breath positives. 7 out of $53(13 \%)$ participants only had virus detected in exhaled breath and not in respiratory secretion samples, whilst 15 out of $53(28 \%)$ only had detectable virus in either their nasal washing or sputum sample.

\section{Comparison of detection of different viruses in respiratory secretions and exhaled}

\section{breath}


In comparison to virus detection in nasal washings, exhaled HRV and RSV were either detected at a markedly lower frequency (1 out of 7) or not at all. In the case of Influenza A, 9 out of 11 nasal washing positives were also positive in exhaled breath. Influenza B also had reasonably high rates of detection with 6 out of 10 nasal washing positives exhibiting a positive response in exhaled breath (Fig. 2A).

Similarly, in comparison to viral detection in sputum, HRV was detected at low levels in only 2 out of 16 participants and again no detection of RSV was observed. Influenza A detection rates were the highest, with more exhaled detection than in sputum (14 out of 12 participants). For Influenza B, 8 out of 12 sputum positives were also exhaled filter positive (Fig. 2B).

The Common Cold Questionnaire (CCQ) assesses viral symptoms on an 11 point scale, and based on the presence or absence of these symptoms, the questionnaire predicts the likelihood of a viral infection. Results are classified into three categories; these are "no virus", "possible virus" or "probable virus" dependent on how many symptoms are reported. 11 out of 53 participants were predicted to have a probable viral infection based on common cold questionnaire responses. Therefore, as 44 of 53 participants had PCR detected virus, it appears that three quarters of viral infections were asymptomatic. 


\section{Discussion:}

The results indicate high levels of viral detection overall, with $83 \%$ of participants showing viral positivity by PCR in the respiratory secretions of all groups tested. Exhaled breath detection correlated strongly with these levels; $81 \%$ of participants who were positive in a respiratory secretion sample also had virus detected on the respiratory filters. This association was strongest for the influenza virus, where $84 \%$ of influenza $\mathrm{A}$ detection in respiratory secretions correlated with exhaled detection. These results indicate high efficacy in the capture of exhaled viruses by the electret filter.

This study demonstrates that spirometry mouthpiece filters are a cheap, easy and noninvasive way to sample exhaled breath for the identification of respiratory viruses. This approach may allow routine sampling of a range of respiratory patients, without the requirement for physicians or lab technicians to be trained in any new techniques.

It is important to note the contribution of asymptomatic carriage, which has been previously shown to be a significant phenomenon in paediatric populations ${ }^{(14,15)}$. Due to the high amount of viral positivity seen when little or no symptoms were reported on the common cold questionnaire, at least some of these patients would simply have had a natural resolution of viral infection without any observable external effect. The impact of asymptomatic infection on chronic respiratory diseases is not yet known, however this study sheds some light on the incidence of it. Longitudinal viral sampling in these cohorts may yield further insights into the development and influence of asymptomatic viral carriage. 
The high levels of viral detection seen may have been attributable to the time of sampling. All patient recruitment and sampling was carried out during a three month time period over June to August. Previous studies have shown ${ }^{(16)}$ that this is the time of greatest overall respiratory viral infectivity in Australia. In NSW, the Australian Influenza Surveillance Report also indicated high levels of influenza A (H3N2) during this time period ${ }^{(17)}$.

A limitation of this study was that nasal washing and sputum samples were obtained from all participants depending on whether the patient was able to spontaneously expectorate sputum. As each of these samples originate from a different area, namely the nose (upper respiratory tract) for nasal washings or from lower in the lungs (lower respiratory tract) for sputum samples, different detection rates are likely to be seen. This may occur due to the tropism of respiratory viruses to either the upper or lower respiratory tract; whilst the ability to produce sputum may indicate different clinical groups of participants. Therefore inaccurate representations of viral presence may have been gained. Also there was a strong differential in the detection of different viruses between the filters and the alternative method. This may reflect the relatively low titre of these in exhaled breath samples, differences in the presence of virus in the upper and lower airways at certain times of infection or difference in the stability of each virus when collected onto the electret of the filter, compared to viral transport medium.

In summary, high levels of virus can be detected across groups of patients with chronic respiratory diseases, often without overt viral symptoms being exhibited. This gives evidence of an important role for viruses within the lung microenvironment and further research may yield insights into contribution of viruses to the disease progression. 
This study has clearly demonstrated the utility of electret filter material contained within spirometry mouthpieces as a novel viral sampling medium to detect exhaled virus. Although detection rates of some respiratory viruses were higher than others, these preliminary findings offer a basis for further research and refinement of this technique.

Future work aims to determine the viability of collected viruses to ensure they are live and able to replicate. Furthermore, we would like to investigate the differences in viral yield between different types of breathing maneuvers. This study focused on patients performing forced expiratory maneuvers as part of normal lung function testing. If this method also worked as well with tidal breathing, it could be applied to an even greater range of patients and also in community viral surveillance.

\section{Acknowledgements:}

I would like to acknowledge the generosity of all the volunteers for providing their time. A special thanks to all the staff of the Respiratory Laboratory at Concord Hospital for their help and to the Cell Biology group at the Woolcock Institute of Medical Research for their contributions. 


\section{$\underline{\text { References: }}$}

1. McManus TE, Marley AM, Baxter N, Christie SN, O'Neill HJ, Elborn JS, et al. Respiratory viral infection in exacerbations of COPD. Respiratory medicine. 2008 Nov;102(11):1575-80.

2. Wedzicha JA, Donaldson GC. Exacerbations of chronic obstructive pulmonary disease. Respiratory care. 2003 Dec;48(12):1204-13; discussion 13-5. 3. Wedzicha JA, Seemungal TA. COPD exacerbations: defining their cause and prevention. Lancet. 2007 Sep 1;370(9589):786-96.

4. (WHO) WHO. Influenza (Seasonal). 2014 [updated March 2014; cited 2015]; Fact Sheet]. Available from:

http://www.who.int/mediacentre/factsheets/fs211/en/.

5. Oshansky CM, Pickens JA, Bradley KC, Jones LP, Saavedra-Ebner GM, Barber $\mathrm{JP}$, et al. Avian influenza viruses infect primary human bronchial epithelial cells unconstrained by sialic acid alpha2,3 residues. PloS one. 2011;6(6):e21183.

6. Mallia P, Johnston SL. Influenza infection and COPD. International journal of chronic obstructive pulmonary disease. 2007;2(1):55-64.

7. Garbino J, Soccal PM, Aubert JD, Rochat T, Meylan P, Thomas Y, et al. Respiratory viruses in bronchoalveolar lavage: a hospital-based cohort study in adults. Thorax. 2009 May;64(5):399-404.

8. Blaschke AJ, Allison MA, Meyers L, Rogatcheva M, Heyrend C, Mallin B, et al. Non-invasive sample collection for respiratory virus testing by multiplex PCR. Journal of clinical virology : the official publication of the Pan American Society for Clinical Virology. 2011 Nov;52(3):210-4. 
9. Heikkinen T, Marttila J, Salmi AA, Ruuskanen O. Nasal swab versus nasopharyngeal aspirate for isolation of respiratory viruses. Journal of clinical microbiology. 2002 Nov;40(11):4337-9.

10. Wurzel DF, Marchant JM, Clark JE, Mackay IM, Wang CY, Sloots TP, et al. Respiratory virus detection in nasopharyngeal aspirate versus bronchoalveolar lavage is dependent on virus type in children with chronic respiratory symptoms. Journal of clinical virology : the official publication of the Pan American Society for Clinical Virology. 2013 Dec;58(4):683-8.

11. Sung RY, Chan PK, Choi KC, Li AM, Nelson EA. A comparative study of nasopharyngeal aspirate and nasal swab specimens for the diagnosis of acute viral respiratory infection. Hong Kong medical journal = Xianggang yi xue za zhi / Hong Kong Academy of Medicine. 2009 Jun;15(3 Suppl 4):24-7.

12. Stelzer-Braid S, Oliver BG, Blazey AJ, Argent E, Newsome TP, Rawlinson WD, et al. Exhalation of respiratory viruses by breathing, coughing, and talking. Journal of medical virology. 2009 Sep;81(9):1674-9.

13. Milton DK, Fabian MP, Cowling BJ, Grantham ML, McDevitt JJ. Influenza virus aerosols in human exhaled breath: particle size, culturability, and effect of surgical masks. PLoS pathogens. 2013 Mar;9(3):e1003205.

14. Jansen RR, Wieringa J, Koekkoek SM, Visser CE, Pajkrt D, Molenkamp R, et al. Frequent detection of respiratory viruses without symptoms: toward defining clinically relevant cutoff values. Journal of clinical microbiology. 2011 Jul;49(7):2631-6. 
15. Jartti T, Lee WM, Pappas T, Evans M, Lemanske RF, Jr., Gern JE. Serial viral infections in infants with recurrent respiratory illnesses. The European respiratory journal. 2008 Aug;32(2):314-20.

16. Murphy B, Phelan PD, Jack I, Uren E. Seasonal pattern in childhood viral lower respiratory tract infections in Melbourne. The Medical journal of Australia. 1980 Jan 12;1(1):22-4.

17. Health AGDo. Australian Influenza Surveillance Report and Activity Updates. 2014 [cited 201527 February]; Available from:

http://www.health.gov.au/flureport. 
Figures:

Table 1: Number of participants showing viral detection in each sample type compared with exhaled detection. A shows sputum samples whilst B shows nasal washings.

\begin{tabular}{|l|l|l|}
\hline $\mathbf{A}=\mathbf{2 3}$ & Sputum positive & Sputum negative \\
\hline Exhaled positive & $14(61 \%)$ & $2(9 \%)$ \\
\hline Exhaled negative & $6(26 \%)$ & \\
& & $1(4 \%)$ \\
& & \\
\hline
\end{tabular}

\begin{tabular}{|l|l|l|}
\hline $\mathbf{B}=\mathbf{3 0}$ & Nasal positive & Nasal negative \\
\hline Exhaled positive & $9(30 \%)$ & \\
& & $5(16.6 \%)$ \\
\hline Exhaled negative & $8(26.7 \%)$ & $8(26.7 \%)$ \\
& & \\
& & \\
\hline
\end{tabular}




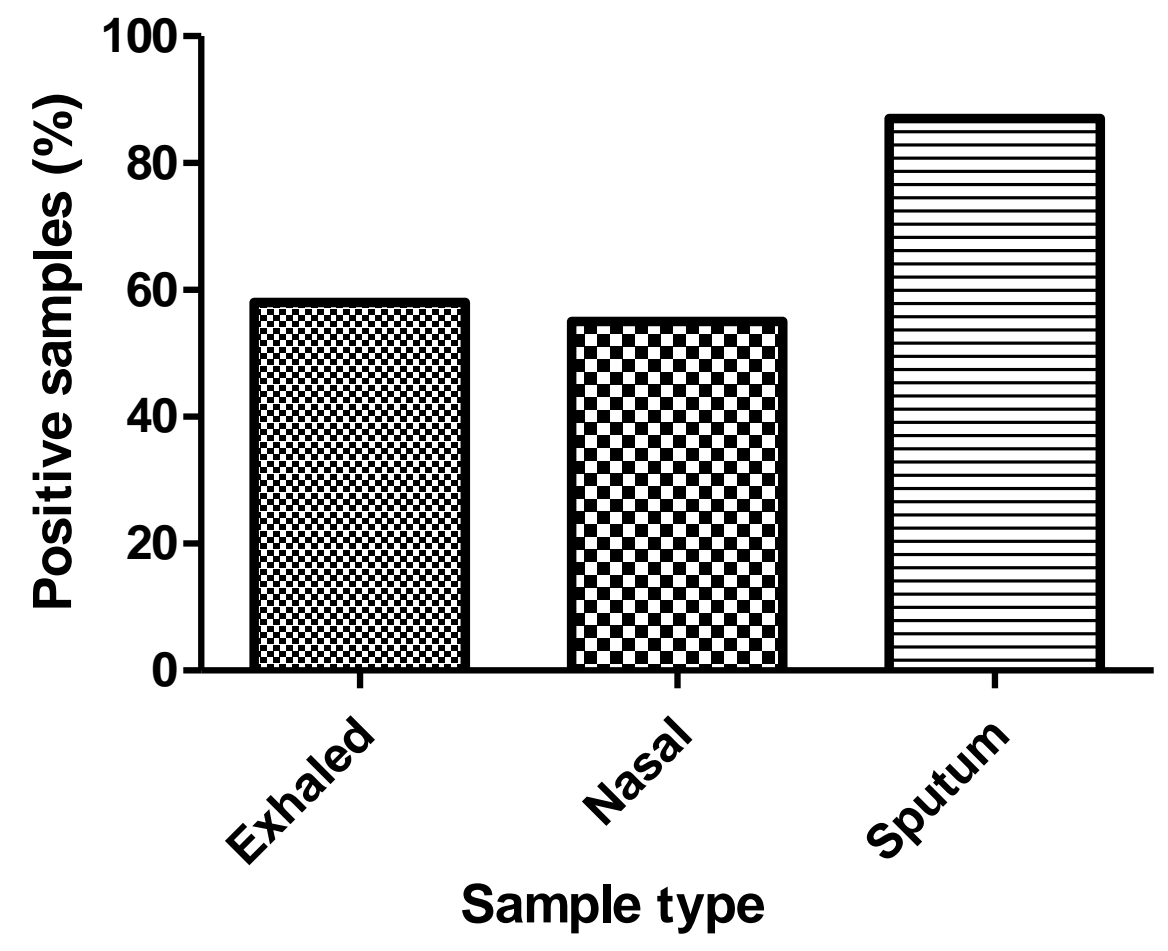

Figure 1: The number of positive viral samples from respiratory secretions and exhaled breath. The percentage of viral positivity was calculated for filter $(n=53)$, nasal washing $(n=30)$ and sputum $(n=23)$ samples from all participants. 
A

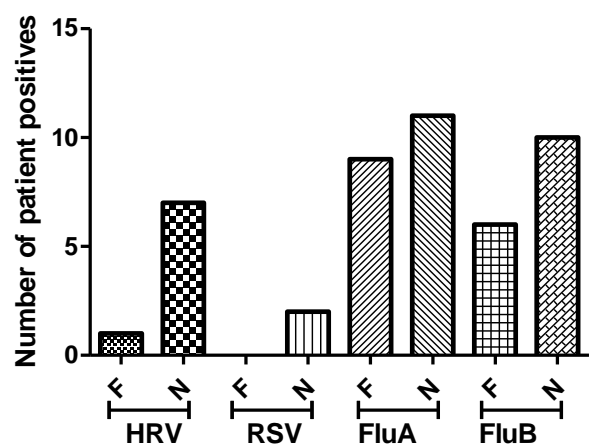

B

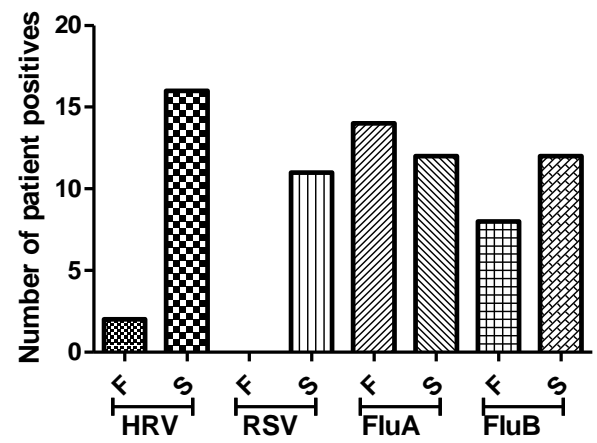

Figure 2: Comparison of virus detection in exhaled breath to either nasal washings or sputum. A shows the comparison of exhaled filter virus detection $(F)$ with detection of virus in nasal washings (N) for each virus. B shows exhaled filter (F) vs. sputum (S) detection levels. 